\title{
Methods of evaluation of autonomic nervous system function
}

Agnieszka Zygmunt, Jerzy Stanczyk

Department of Paediatric Cardiology, Medical University of Lodz, Poland

Submitted: 3 September 2009

Accepted: 3 December 2009

Arch Med Sci 2010; 6, 1: 11-18

DOI 10.5114/aoms.2010.13500

Copyright $\odot 2010$ Termedia \& Banach

\section{Abstract}

Disturbances of the autonomic nervous system play a crucial role in the pathogenesis and clinical course of many diseases. Recently, rapid development has occurred in the clinical assessment of autonomic function. Various procedures have been described as diagnostic tools to monitor autonomic dysfunction. Some of them are mostly used for research purposes. Many, however, have found their place in routine clinical evaluation. Our paper presents selected methods of assessment of the autonomic nervous system with particular emphasis on those that are useful in diagnosis and treatment of diseases of the cardiovascular system. We discuss multiple tests based on cardiovascular reflexes, methods of studying heart rate variability as well as direct catecholamine measurements. Moreover, we outline tests of sudomotor function and microneurography.

Key words: sympathetic activity, parasympathetic activity, assessment.

\section{Introduction}

The autonomic nervous system is the part of the nervous system that is responsible for regulation and integration of internal organs' functioning. Together with the endocrine and immunological systems it determines the status of the internal environment of the organism and adjusts it to its current needs, thus enabling adaptation of the internal environment to changes in the external environment [1, 2]. Disorders of autonomic regulation are described in multiple and diverse diseases, both those that directly afflict the nervous system as well as those afflicting other organs, where they trigger or enhance pathological symptoms [3-10]. In the past decades, the significance of disturbances of autonomic regulation in circulatory system diseases has been especially emphasized [11-19]. Clinical symptoms of these disturbances are frequently non-characteristic and therefore in order to identify them it is essential to know the methods of a more detailed assessment of autonomic nervous system function. Many methods evaluating the autonomic nervous system have been described. Some of them are applied in clinical practice while others are used mostly in scientific studies $[3,6,9,20-25]$. Our paper presents selected methods of assessment of the autonomic nervous system with particular emphasis on those that are useful in diagnosis and treatment of diseases of the cardiovascular system.

\author{
Corresponding author: \\ Agnieszka Zygmunt, PhD \\ Department of Paediatric \\ Cardiology \\ Medical University \\ of Lodz \\ 36/50 Sporna \\ 91-738 Lodz, Poland \\ Phone/fax: +48 426177700 \\ E-mail: azygmunt@tlen.pl
}




\section{Conditions of autonomic system evaluation}

Multiple factors influence autonomic function body position, emotional state, ingested food and medicines, as well as other substances [6]. Caffeine and nicotine should be withheld for at least 3-4 h before testing, alcohol for $8 \mathrm{~h}$. If possible, sympathomimetic drugs should be stopped for 24-48 h before testing, and anticholinergics for $48 \mathrm{~h}$ [23]. Moreover, standardization of test conditions is crucial in order to make them comparable, especially during the assessment of cardiovascular reflexes. Directly before testing, the patient should be laid down or seated for about 30 min in a quiet room with neutral temperature and humidity [23].

\section{Tests assessing autonomic function}

Most of the tests are based on evaluation of the cardiovascular reflexes triggered by performing specific provocative manoeuvres. Stimuli that raise blood pressure, such as isometric exercise, cold pressor test or mental arithmetic, activate mainly sympathetic outflow. Moreover, blood pressure responses to orthostatic testing and Valsalva manoeuvre are in a large part a reflection of sympathetic activity $[10,21,23,24]$. Changes in heart rate during orthostatic testing and Valsalva manoeuvre, as well as during deep breathing or diving reflex, reflect parasympathetic modulation $[10,21,24]$.

Given the complexity of the autonomic system there is no single test that precisely reflects function of a specific branch of this system. Therefore, it is not uncommon to order numerous tests based on diverse reflexes. Traditionally, batteries of autonomic tests have been introduced, with the Ewing battery being the most popular. It is widely used in diagnosis of diabetic neuropathy and it comprises Valsalva manoeuvre, response to deep breathing, orthostatic testing and isometric exercise [1]. More recently, new techniques, such as evaluation of heart rate variability or microneurography, have been introduced as diagnostic tools. Virtually each medical specialty has worked out its own battery of tests in order to assess those aspects of autonomic functioning that are most relevant in a specific field.

\section{Tests of autonomic cardiovascular reflexes}

\section{Valsalva manoeuvre}

Valsalva manoeuvre evaluates function of baroreceptors [21, 23, 26]. It is a voluntary forced expiration of a subject against a resistance. An increase in transthoracic pressure mechanically leads to transient increase in blood pressure (phase I), which, by activation of baroreceptors, simultaneously results in a slight bradycardia. Then, due to limited venous return and low stroke volume, blood pressure decreases with concomitant compensatory tachycardia (phase II). When the expiration is stopped (phase III), a further transient fall in blood pressure is observed because of pulmonary vasculature expansion, while heart rate increases. In phase IV, probably due to baroreceptors' activation, an abrupt rise in blood pressure above the initial values with concomitant bradycardia occurs. Low-pressure mechanoreceptors are not significantly engaged during this manoeuvre. Based on changes in haemodynamic parameters, various indices can be calculated. Valsalva ratio is the most important of them and it is derived from the longest RR interval in phase IV divided by the shortest RR interval in phase II and at the very beginning of phase III [6, 24]. Its value below 1.21 is considered abnormal [1, 2, 27]. Valsalva ratio reflects parasympathetic activity [6], whereas alterations in blood pressure are a measure of sympathetic function - its fall at the beginning of phase II should not exceed $21 \mathrm{mmHg}$ and at the end of phase II or in phase III it should return to baseline values [23].

The manoeuvre is performed with the subject seated, after 15-20 min' rest. He or she is then asked to blow into a special tube to maintain a column of mercury at $40 \mathrm{mmHg}$ for $15 \mathrm{~s}$. A clamp is placed on the nose and it is suddenly released after $15 \mathrm{~s}$. ECG is recorded during the resting period and during the subsequent 40 heart beats after releasing the clamp. The manoeuvre is generally performed three times and the mean value is calculated [24]. Some authors suggest taking into account the highest value [6].

It should be taken into consideration that some patients may perform the Valsalva manoeuvre improperly as they raise only mouth pressure without increasing intrathoracic pressure, which may result in falsely abnormal changes in RR intervals [10]. Apart from the manner of performing the test, its results may be affected by age, sex, body position and medications taken [23].

\section{Deep breathing}

This test is based on the phenomenon of respiratory arrhythmia, which is most pronounced at the respiration rate of 6 breaths per minute. The subject is asked to breathe at this rate (with $5 \mathrm{~s}$ of inhalation and $5 \mathrm{~s}$ of exhalation per breath), and then the difference between the average of the largest accelerations during inspiration and the average of the largest decelerations during expiration is calculated. It should not be lower than $10-15$ beats per minute [1, 20, 21, 27]. Respiratory arrhythmia declines with age. After the age of 50 this difference should be at least 5 beats per minute [20].

The expiratory-inspiratory ratio ( $\mathrm{E}:$ I ratio), which is the ratio of the longest $R R$ interval during 
expiration and the shortest RR interval during inspiration from 5 cycles, can also be determined. The $E$ : I ratio in young persons should be higher than $1.2[20,24]$.

Results of this test are affected by age, resting heart rate, body mass index and administered medications [23].

\section{Isometric handgrip test}

A rise in diastolic blood pressure is determined during isometric pressing of a handgrip dynamometer at approximately one third of the maximum contraction strength for 3-5 min. Blood pressure measurements are taken at the other arm at 1 min interval. An increase in diastolic blood pressure is a result of heart rate acceleration without an increase of peripheral vascular resistance. The test result is presented as the difference between the highest diastolic pressure during the examination and the average diastolic pressure at rest. It should normally be higher than $15 \mathrm{mmHg}[1,27]$.

Special attention should be paid to the manner of performing the handgrip by the subject as many patients perform a Valsalva manoeuvre during this test and consequently bias its results [20].

\section{Cold pressor test}

Immersion of hands or feet for about 60-90 s in cold water $\left(4^{\circ} \mathrm{C}\right)$ should, due to activation of afferent pain and temperature fibres from the skin as well as emotional arousal, lead to sympathetic activation and increase in blood pressure and heart rate $[10,24]$. A rise in diastolic blood pressure is calculated and it should normally exceed $15 \mathrm{mmHg}$.

A different kind of response takes place after immersion of the face in water with breath holding (diving reflex), as regardless of water temperature it leads to bradycardia [1,27]. Heart rate decreases by approximately 40 beats per minute, while blood pressure increases by approximately $25 \mathrm{mmHg}$. A cold face test is a modification of the latter procedure during which a cold compress $\left(1-2^{\circ} \mathrm{C}\right)$ is applied to the subject's face for a period of 1-3 min. It has similar sensitivity in evaluating parasympathetic response. However, it is better tolerated by the patients. Moreover, it may be performed in subjects who are unable to cooperate with other challenge manoeuvres [20].

\section{Mental arithmetic}

This test is based on performing serial subtraction (usually 100 minus 7 or 1000 minus 13) which aims at activating sympathetic outflow. The subsequent increase in systolic blood pressure should exceed $10 \mathrm{mmHg}[1,27]$.

\section{Active standing (orthostatic test)}

Haemodynamic responses to active standing are assessed during this test. Prior to the manoeuvre, the subject is rested in a supine position. Directly after assumption of the upright position a substantial amount of blood is redistributed to blood vessels of the lower extremities, which decreases venous return and cardiac stroke volume. In order to maintain adequate values of haemodynamic parameters, the organism initiates a physiological compensatory reaction. It can be divided into an immediate response with an abrupt fall in systolic and diastolic blood pressure and a visible acceleration of heart rate (first $30 \mathrm{~s}$ ), a phase of early stabilization, which occurs after approximately 1-2 min, and a response to prolonged orthostasis lasting for more than 5 min. Introduction of mechanisms compensating gravitational blood redistribution results during the phase of stabilization in acceleration of heart rate by about 10-15 beats per minute and a slight decrease in systolic blood pressure, while diastolic pressure increases by approximately $10 \mathrm{mmHg}[20,24,28]$.

Evaluation of changes in heart rate (30/15 ratio) is performed during the initial phase of adaptation to orthostasis (first $45 \mathrm{~s}$ ), and the ratio is calculated as a quotient of the maximal (around $30^{\text {th }}$ heart beat) to minimal (near $15^{\text {th }}$ heart beat) RR interval in this period. The $30 / 15$ ratio should be at least 1.04, but it decreases with age [21].

Fluctuations of blood pressure are assessed based on somewhat later responses to standing (first $4 \mathrm{~min}$ ), and they are expressed as the difference between the baseline supine and the minimal blood pressure after standing up. A decline in systolic blood pressure by more than $20 \mathrm{mmHg}$ and by more than $10 \mathrm{mmHg}$ for diastolic blood pressure is considered abnormal [1, 23, 27].

Active standing is more suited than a head-up tilt test to assess responses during the initial phase of orthostatic challenge. It is the best test to diagnose idiopathic orthostatic hypotension [20].

\section{Head-up tilt test}

The test evaluates adaptation to orthostasis and subsequent changes in haemodynamic parameters after passive tilting on a special motorized table. The differences in the haemodynamic response compared with active standing are seen only during the initial period. Thanks to passive and gradual assumption of the upright posture, abrupt fluctuations of blood pressure and heart rate, characteristic of active standing up, are not present. The head-up tilt test is a suitable diagnostic tool for assessment of autonomic regulation during longduration orthostatic challenge, and thus it has become a key element in the diagnosis of 
neurocardiogenic syncope. Its sensitivity in this aspect exceeds the sensitivity of an orthostatic test since there is a contraction of abdominal and leg muscles during active standing, which exerts a pumping influence on blood vessels, which in turn decreases peripheral blood pooling $[6,20]$.

A provocative tilt test is performed on an automated tilt test table, which allows a consistent slow tilt to 60-80 degrees. It should be carried out in the morning after the overnight fast or several hours after a meal. In the first phase the patient remains supine for a period of about 15-30 min. ECG and blood pressure recordings are obtained prior to the test and during 30-45 min of tilting. In some laboratories, apart from gravitational provocation, an additional pharmacological provocation is used (mostly intravenous infusion of isoproterenol or sublingual nitroglycerine) [29, 30, 31, 32]. It increases the test sensitivity and allows for a shorter period of time necessary to achieve a positive end point. However, it decreases the test specificity. The tilt angle of 60 degrees seems to be optimal, as at this point $90 \%$ of the gravitational blood volume displacement occurs in comparison to a fully upright position, while abdominal and lower extremities muscle engagement is not yet observed $[6,20]$. In subjects with a positive tilt test result the syncope generally takes place between the $10^{\text {th }}$ and $30^{\text {th }} \mathrm{min}$ of examination. There are several pathological responses to tilt-table testing [33]. A classic vasovagal (neurocardiogenic) response with a sudden decrease in blood pressure and heart rate is the most frequent. It can be subdivided into three subsets - vasodepressor, cardioinhibitory or mixed, based on the relative predominance of hypotension or bradycardia [33]. Postural tachycardia syndrome (PTS) is a different type of pathological response, which is characterized by excessive acceleration of heart rate aimed at compensating decreased peripheral vascular resistance. Less frequently met responses include dysautonomic pattern with a gradual fall in blood pressure or a psychogenic reaction.

Sometimes, in order to diagnose noncharacteristic syncope (postprandial, exercise induced), the head-up tilt test is combined with another provocative stimulus such as a balanced liquid meal or a modified exercise test [10]. The tilt test procedure is also used in the process of treatment of syncopal patients [34].

\section{Baroreflex sensitivity testing}

Traditional approaches to testing the sensitivity of baroreceptors of the carotid sinus and aortic arch include pharmacological stimulation and neck suction. In order to stimulate baroreceptors a subject is administered intravenous phenylephrine, either in a bolus or less frequently in constant infusion. A rise in blood pressure generates bradycardia, and changes of systolic blood pressure and RR intervals are presented in the form of a special graph. The baroreflex sensitivity is calculated as the slope of the linear regression between beat-to-beat systolic blood pressure values and the values of the RR interval, which normally should be above $10 \mathrm{~ms} / \mathrm{mmHg}$ [1]. Evaluation of baroreflex sensitivity is also performed by their decompression, which is achieved by administration of nitroglycerine or sodium nitroprusside. However, interpretation of obtained results is more difficult with this method [1].

Standard methods of baroreceptor stimulation also include application of negative pressure to the neck by means of a mouldable lead collar. In contrast to pharmacological activation of the baroreflex, neck chamber stimulation determines not only heart rate responses to baroreceptor stimulation but also blood pressure responses [20]. Sometimes, a carotid sinus massage with assessment of the subject's response to direct, mechanical activation of baroreceptors is performed. During this procedure the patient may sit or lie down and the sinus stimulation lasts between 5 and $10 \mathrm{~s}$ [30]. In suspected carotid sinus hypersensitivity, resuscitation facilities should be available as carotid massage may cause profound bradycardia or cardiac arrest [10].

Advances in beat-by-beat blood pressure and heart rate monitoring methods have now made it possible to estimate non-invasively the baroreflex sensitivity from the RR interval changes associated with spontaneous fluctuations in blood pressure. It has been demonstrated that results of such analysis are closely correlated with the results of the phenylephrine method. This new methodology does not require patient cooperation. However, it only evaluates the spontaneous baroreflex sensitivity under resting conditions, and provides no information about modulation of blood pressure in response to baroreceptor activation.

The so-called sequence method analyzes the relations between fluctuations in blood pressure extracted in particular sequences and RR intervals. In this technique, beat-by-beat blood pressure and RR interval recordings are scanned for sequences in which systolic blood pressure and RR interval concurrently increase or decrease for at least three consecutive beats. Baroreflex sensitivity is then assessed from the relationship between systolic blood pressure and RR interval across these fragments. A second method provides a baroreflex estimate based on spectral analysis of heart rate and blood pressure variability. With this method, baroreflex sensitivity is assessed by analyzing the RR interval changes associated with rhythmic blood 
pressure oscillations over a range of frequencies reported to reflect baroreflex function [11, 20].

\section{Analysis of heart rate variability}

Analysis of heart rate variability (HRV) has nowadays become one of the most popular methods of autonomic nervous system evaluation. It is based on the observation that even at rest the duration of RR intervals is not constant but continually fluctuates around the mean value. Extremely complex neural mechanisms are responsible for these fluctuations. They are based mainly on interactions between the sympathetic and parasympathetic nervous system. It is noteworthy that some of the autonomic neurons and fibres, apart from the transient phasic activity, initiated by particular stimuli, demonstrate basal tonic activity with characteristic rhythmic fluctuations whose frequency differs for different neurons and fibres: from high-frequency oscillations characteristic of heart or respiratory rate to extremely slow oscillations whose rhythm is circadian, monthly or even seasonal [2]. Blood pressure is another haemodynamic parameter whose variability, besides heart rate, is also assessed in clinical practice [1]. Activity of the sympathetic and parasympathetic nervous system continually fluctuates, which results from mutual interactions. At rest, vagal activity dominates and it is mainly responsible for heart rate variability. Therefore, sinus arrhythmia, which is vagally mediated, is the most prominent variability at rest. The influence of parasympathetic activation is quick and transient due to fast acetylcholine degradation by acetylesterase. Because of this, effects of parasympathetic neuron excitation are visible in the next cycle after the stimulus and the parasympathetic nervous system accounts for quick changes in heart rate. Sympathetic stimulation develops more slowly and its effects are visible as a change in rhythm after 2-3 s; thus it is responsible for slower oscillations but of higher amplitude. Moreover, it is limited by vagal activity with mutual modulations [1, 35, 36]. During the evaluation of HRV indices one must realize that they are not direct indices of tonic activity of the sympathetic and parasympathetic nervous system, but rather the resultant of their influence on the effectors, which are the receptors of sinus node cells.

Heart rate variability is generally assessed based on time-domain or frequency-domain analysis. Indices of time-domain analysis derive from either direct RR interval measurements or the differences between successive RR intervals. Most frequently, they are calculated over a full 24-h ECG recording. Sometimes, they are calculated over shorter, e.g. 5-min, recordings in order to evaluate the influence of various factors on HRV [1, 35-37].
Parameters of time-domain analysis include:

1) SDNN (the standard deviation of NN, so-called normal-to-normal, intervals - it encompasses both long- and short-term variability and thus describes the overall HRV),

2) SDANN (the standard deviation of the average NN intervals calculated over successive 5-min periods of 24-h recording - it evaluates slow changing components of HRV),

3) rMSDD (the square root of the mean squared differences of successive NN intervals - it describes short-term variation and thus reflects parasympathetic activity), and

4) pNN50 (the proportion of differences in consecutive NN intervals that are longer than $50 \mathrm{~ms}$ - its significance is similar to rMSDD).

Spectral analysis does not expresses heart rate as a function of time but as a function of frequency. It concentrates on revealing the cyclical nature hidden in the series of changing RR intervals. The frequency and magnitude of these oscillations are measured, which allows the calculation of the power density for separate frequency ranges. It is recommended to calculate the frequency-domain indices over short-term recordings, preferably 5-min ones [ $1,35,36]$. In most studies two components are assessed:

1) LF (component of the low-frequency range, $0.04-0.15 \mathrm{~Hz}$, modulated by both the sympathetic and parasympathetic nervous system and also associated with baroreceptor activity),

2) HF (component of the high-frequency range, 0.15-0.4 Hz, modulated by the parasympathetic nervous system, connected with respiration and blood pressure changes), and

3) LF/HF ratio, reflecting interactions of both types of autonomic modulation.

The component of the very low frequency (VLF) range (below $0.04 \mathrm{~Hz}$ ) is estimated less often since its interpretation in short recordings ( 5 min or less) is unclear. Therefore, it is not recommended to interpret the VLF component derived from such fragments. Over longer periods it probably reflects the activity of baroreceptors [35, 36].

Recently, analysis of HRV by methods based on non-linear dynamics has been introduced [38, 39]. However, their application is still limited to scientific research. Although the frequency-domain analysis of HRV is much better understood, it is also mostly used for research purposes. Time-domain HRV analysis has the widest application in routine clinical evaluation and some of its indices have become well-documented, independent risk factors of cardiovascular events.

Heart rate variability changes significantly with age. Moreover, it may be influenced by other factors such as body position, physical and mental activity, body mass index, sex or even diet [6, 20, 25, 35, 40]. 


\section{Measurements of neurotransmitter levels}

The measurement of circulating catecholamines and other substances that are modulated by the autonomic nervous system belongs to classic methods assessing its function [6]. Activity of the sympathetic nervous system is evaluated based on the plasma or urine noradrenaline (NA) concentration. Such measurements, however, have significant limitations, as NA is subjected to changeable reuptake dependent on the density of the basilar plexus and blood flow velocity in a specific organ. Moreover, circulating NA represents only a small fraction (5-10\%) of the amount of neurotransmitter secreted from nerve terminals [11].

The measurement of plasma NA is, however, an improvement over the assessment of urine adrenaline (A), NA and their precursors and metabolites, which were traditionally used to evaluate the autonomic nervous system tone [11].

Plasma A provides a measure of adrenal medullary activity. The value of plasma catecholamines measurement is increased if it is combined with assessment of responses to adrenergic antagonists and agonists (phenylephrine, tyramine and isoproterenol are used among other substances) or if changes in neurotransmitter levels are evaluated after various physiological stimuli such as standing. It has been found that in healthy subjects NA concentration should increase by approximately $100 \%$ after $5-10$ min of orthostatic challenge $[10,21]$.

Acetylcholine is very labile, as it is quickly disintegrated by acetylcholinesterase, and it cannot be precisely quantified [2].

\section{Noradrenaline spillover rate}

This method permits assessment of NA release from specific target organs. The NA radiolabelled method is based on intravenous infusion of small amounts of tritiated NA, which allows tissue clearance of this substance to be subtracted from plasma NA values and the remainder to be made a marker of the neurotransmitter "spillover" from neuroeffector junctions. This "spillover" in steadystate conditions mirrors the secretion of NA from the sympathetic nerve terminals [11].

Invasive techniques measure not only total body but also regional NA spillover in the heart, splanchnic and renal circulation, and the brain [10].

\section{Other radioisotope techniques}

Cardiac sympathetic innervation can be assessed by means of radionuclide 123-meta-iodobenzylguanidine [10].

\section{Microneurography}

This method utilizes the presence of postganglionic sympathetic fibres as separate anato- mical fascicles in mixed somatic nerves going to muscles. Sympathetic nerve activity from large, peripheral nerves located superficially under the skin (usually the peroneal, tibial or median nerve), is directly recorded by means of tungsten microelectrodes inserted selectively into muscle or skin fascicles [2].

Microneurography permits separate recordings of sympathetic nerve activity to muscle (MSNA) or skin (SSNA) vessels. Muscle sympathetic nerve activity reflects the vasoconstrictor signal to the skeletal muscle vasculature. It is highly sensitive to blood pressure changes and is regulated by means of baroreflexes, both arterial and cardiopulmonary. These reflexes do not affect SSNA. SSNA reflects vasomotor neural traffic to skin blood vessels with almost no sudomotor activity. The two recordings (MSNA and SSNA) differ significantly with regard to morphology. Studies to date have shown that measurement of sympathetic nerve activity from peripheral nerves is safe, accurate and reproducible. Furthermore, it has been proved that recordings from one limb can be reliably assumed to reflect recordings of sympathetic nerve activity to the muscle vascular bed throughout the body. The method's quantitative nature is also a significant advantage [11].

\section{Testing of sudomotor function}

The cholinergic part of the autonomic nervous system can be assessed based on sweat glands' reaction to various stimuli. They are important effectors of thermoregulation in humans, and their activity is stimulated by impulses conducted by cholinergic postganglionic sympathetic fibres.

\section{Thermoregulatory sweat test}

In this test, sweat secretion after raising body temperature by $1-1.4^{\circ} \mathrm{C}$ (but not above $38^{\circ} \mathrm{C}$ ) is measured. Various indicators, mostly powders (quinizarine or alizarin red) that change colour on exposure to moisture, are used [10]. The subject is placed in a sweat chamber with air temperature at $45-50^{\circ} \mathrm{C}$ and humidity at $35-50 \%$. The peak response of sweat glands occurs after 35-45 min. Normal subjects should demonstrate generalized perspiration [24].

\section{Sympathetic skin response (SSR)}

The test is based on the temporary change in skin electrical resistance in response to activation of sweat glands by various endogenous and exogenous stimuli [24]. This method measures electrical potentials from electrodes situated on the palms of the hands and soles of the feet, which reflect sympathetic cholinergic activity of sweat glands. The stimuli used are physiological (loud 
noise, flash, touch, inspiratory gasps) or electrical (peripheral nerve stimulation - median, tibial, peroneal, supraorbital nerve) [10].

\section{QSART (Quantitative Sudomotor Axon Reflex Test)}

This test assesses the sudomotor nerve fibres by means of direct activation using the axon reflex (acetylcholine iontophoresis). A sudorometer (a device that quantitates sweat volume) and multicompartment sweat capsules attached to the skin at 4 different sites are needed to perform the test. Separate compartments of the capsule allow not only the sweat glands to be stimulated (acetylcholine iontophoresis) but also the response to be recorded (measurement of skin humidity) [24]. This is the most precise test evaluating thermoregulation.

\section{Conclusions}

Assessment of autonomic function is difficult and time-consuming. It requires precision and experience. It is extremely important to appropriately standardize conditions of the tests. The patient must be carefully prepared in order to stabilize haemodynamic parameters and then the test protocol needs to be strictly observed. Only then can reproducibility of specific tests be guaranteed. When continuous blood pressure monitoring is required during any of the tests, one should avoid invasive methods and substitute them with new, non-invasive techniques such as Finapres or arterial tonometry. Textbooks and publications provide approximate reference value ranges. However, some authors point out that for some tests control values should be established separately for each laboratory.

Patients may consider some of the described tests unpleasant (e.g. cold pressor test, neck collar, microneurography) and they may be reluctant to participate in a second test run.

Despite all these difficulties, knowledge of the tests assessing autonomic function is extremely important in clinical practice. Wider implementation of these techniques, especially those that are the most objective, together with their competent interpretation may contribute to a better understanding of the role of the autonomic nervous system in pathogenesis of many diseases and translate into better patient care.

\section{Acknowledgments}

The study was supported by a grant from the Medical University of Lodz (no. 502-18-674).

\section{References}

1. Prusiński A, Rozentryt P. Odruchy autonomiczne. In: Traczyk WZ (ed). Diagnostyka czynnościowa człowieka [Polish].
Fizjologia stosowana. PZWL, Warszawa 1999; 213-27.

2. Trzebski A. Autonomiczny układ nerwowy i mięśni gładkie. In: Traczyk WZ, Trzebski A (eds). Fizjologia człowieka z elementami fizjologii stosowanej i klinicznej [Polish]. PZWL, Warszawa 2001; 508-88.

3. Low PA. Testing the autonomic nervous system. Semin Neurol 2003; 23: 407-21.

4. Carnethon MR, Prineas RJ, Temprosa M, Zhang ZM, Uwaifo G, Molitch ME; Diabetes Prevention Program Research Group. The association among autonomic nervous system function, incident diabetes, and intervention arm in the Diabetes Prevention Program. Diabetes Care 2006; 29: 914-19.

5. Vinik Al, Maser RE, Mitchell BD, Freeman R. Diabetic autonomic neuropathy. Diabetes Care 2003; 26: 1553-79.

6. Scott WA. Assessment of the autonomic nervous system. In: Moss and Adams heart disease in infants and adolescents including the fetus and young adult. Baltimore (et.) Williams and Wilkins 1995; 172-81.

7. Tanaka $\mathrm{H}$. Autonomic function and child chronic fatigue syndrome. (Abstract) Nippon Rinsho 2007; 65: 1105-12.

8. El-Sayed HL, Kotby AA, Tomoum HY, El-Hadidi ES, El Behery SE, El-Ganzory AM. Non-invasive assessment of cardioregulatory autonomic functions in children with epilepsy. Acta Neurol Scand 2007; 115: 377-84.

9. Klein CM. Evaluation and management of autonomic nervous system disorders. Semin Neurol 2008; 28: 195-204.

10. Mathias CJ. Autonomic diseases: clinical features and laboratory evaluation. J Neurol Neurosurg Psychiatry 2003; 74 (Suppl 3): iii31-41.

11. Sinski M, Lewandowski J, Abramczyk P, Narkiewicz K, Gaciong Z. Why study sympathetic nervous system? J Physiol Pharmacol 2006; 57 (Suppl. 11): 79-92.

12. Mansoor GA. Orthostatic hypotension due to autonomic disorders in the hypertension clinic. Am J Hypertens 2006; 19: 319-26.

13. Medow MS, Steward JM, Sanyal S, Mumtaz A, Sica D, Frishman WH. Pathophysiology, diagnosis and treatment of orthostatic hypotension and vasovagal syncope. Cardiol Rev 2008; 16: 4-20.

14. Lahiri MK, Kannankeril PJ, Goldberg JJ. Assessment of autonomic function in cardiovascular disease: physiological basis and prognostic implications. J Am Coll Cardiol 2008; 51: 1725-33.

15. Zygmunt A, Stanczyk J. Heart rate variability in children with neurocardiogenic syncope. Clin Auton Res 2004; 14: 99-106.

16. Rabbia F, Silke B, Carra R, et al. Heart rate variability and baroreflex sensitivity during fosinopril, irbesartan and atenolol therapy in hypertension. Clin Drug Investig 2004; 24: 651-59.

17. Nolan J, Batin PD, Andrews R, et al. Prospective study of heart rate variability and mortality in chronic heart failure: results of the United Kingdom heart failure evaluation and assessment of risk trial (UK-heart). Circulation 1998; 98: 1510-6.

18. Mathias CJ. Role of autonomic evaluation in the diagnosis and management of syncope. Clin Auton Res 2004; 14 (Suppl 1): 45-54.

19. Kleiger RE, Miller JP, Bigger JT, Moss AJ; Multicenter PostInfarction Research Group. Decreased heart rate variability and its association with increased mortality after acute myocardial infarction. Am J Cardiol 1987; 59: 256-62.

20. Hilz ML, Dütsch M.Quantitative studies of autonomic function. Muscle Nerve 2006; 33: 6-20. 
21. Taylor AA, Marcus B. Interaction of the nervous system and the heart. In: The science and practice of pediatric cardiology. Garson A, Bricker JT Jr, Fisher DJ, Neish SR. Williams and Wilkins 1998; 415-42.

22. Junqueira LF Jr. Teaching cardiac autonomic function dynamics employing the Valsalva (Valsalva-Weber) maneuver. Adv Physiol Educ 2008; 32: 100-6.

23. Jaradeh SS, Prieto TE. Evaluation of the autonomic nervous system. Phys Med Rehabil Clin N Am 2003; 14: 287-305.

24. Pierzchała K, Łabuz-Roszak B. Wybrane metody oceny autonomicznego układu nerwowego [Polish]. Wiad Lek 2002; 55: 325-31

25. Lambert E, Straznicky N, Eikelis N, et al. Gender differences in sympathetic nervous activity: influence of body mass and blood pressure. J Hypertens 2007; 25: 1411-9.

26. Zöllei E, Paprika D, Rudas L. Measures of cardiovascular autonomic regulation derived from spontaneous methods and the Valsalva maneuver. Auton Neurosci 2003; 103: 100-5.

27. van den Berg MP, Smit AJ. Bedside autonomic function testing in patients with vasovagal syncope. Pacing Clin Electrophysiol 1997; 20: 2039-42.

28. Grubb BP. Pathophysiology and differential diagnosis of neurocardiogenic syncope. Am J Cardiol 1999; 84: 3Q-9Q.

29. Zygmunt A, Stańczyk J. Zastosowanie testu pionizacyjnego w diagnostyce omdleń u dzieci i młodzieży [Polish]. Pol Przegl Kardiol 2005; 3: 249-53.

30. D33 European Society of Cardiology. Task Force Report. Guidelines on management (diagnosis and treatment) of syncope. Eur Heart J 2001; 22: 1256-306.

31. Grubb BP, Kimmel S. Test pionizacyjny. Bezpieczna i prosta metoda diagnostyczna w przypadku omdleń neeurokardiogennych [Polish]. Med po Dypl 1999; 8: 119-24.

32. American College of Cardiology. Expert Consensus Document. Tilt table testing for assessing syncope. J Am Coll Cardiol 1996; 28: 263-75.

33. Grubb BP Neurocardiogenic syncope. In: Grubb BP, Olshansky B (eds). Syncope. Futura Publishing Company 1998; 73-106.

34. Kozłowski D, Łepska L, Zapaśnik P, et al. The estimation of clinical and home-based tilt training efficacy. Arch Med Sci 2007; 3: 351-54.

35. European Society of Cardiology and the North American Society of Pacing and Electrophysiology. Special Report. Heart rate variability. Standards of measurements, physiological interpretation and clinical use. Circulation 1996; 93: 1043-65.

36. Piotrowicz R, Baranowski R, Dąbrowski A, et al. Zmienność rytmu zatokowego [Polish]. ESS 1998; 5 (Suppl. II): 30-4.

37. Pinna GD, Maestri R, Torunski A, et al. Heart rate variability measures: a fresh look at reliability. Clin Sci (Lond) 2007; 113: 131-40.

38. Maestri R, Pinna GD, Porta A, et al. Assessing nonlinear properties of heart rate variability from short-term recordings: are these measurements reliable? Physiol Meas 2007; 28: 1067-77.

39. Makikallio TH, Tapanainen JM, Tulppo MP, Huikuri HV. Clinical applicability of heart rate variability analysis by methods based on nonlinear dynamics. Card Electrophysiol Rev 2002; 6: 250-5.

40. Weisinger HS, Salem N Jr, Makino KK, et al. Effect of dietary omega-3 fatty acid deficiency on heart rate variability in hooded rats. Arch Med Sci 2007; 3: 208-14 